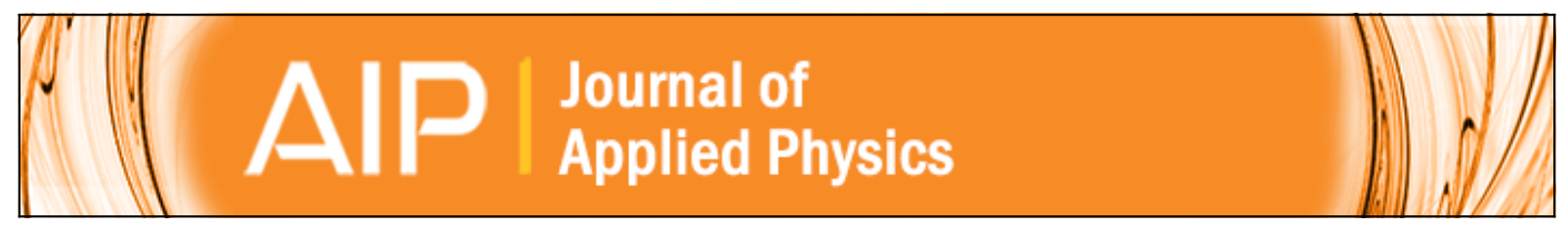

\title{
Control of thermal runaway in microwave resonant cavities
}

X. Wu, J. R. Thomas Jr., and W. A. Davis

Citation: Journal of Applied Physics 92, 3374 (2002); doi: 10.1063/1.1501744

View online: http://dx.doi.org/10.1063/1.1501744

View Table of Contents: http://scitation.aip.org/content/aip/journal/jap/92/6?ver=pdfcov

Published by the AIP Publishing

\section{Articles you may be interested in}

Experimental evidence of redistribution of fields during processing in a high-power microwave cavity

Appl. Phys. Lett. 85, 3632 (2004); 10.1063/1.1806542

Method of determining the wall conductivity of a multimode microwave applicator

Rev. Sci. Instrum. 75, 2678 (2004); 10.1063/1.1775315

Development of a high power microwave plasma beam applicator

Rev. Sci. Instrum. 72, 4273 (2001); 10.1063/1.1406920

Thermal profiles and thermal runaway in microwave heated slabs

J. Appl. Phys. 85, 3774 (1999); 10.1063/1.369747

Thermal runaway in microwave heated isothermal slabs, cylinders, and spheres

J. Appl. Phys. 83, 438 (1998); 10.1063/1.366657

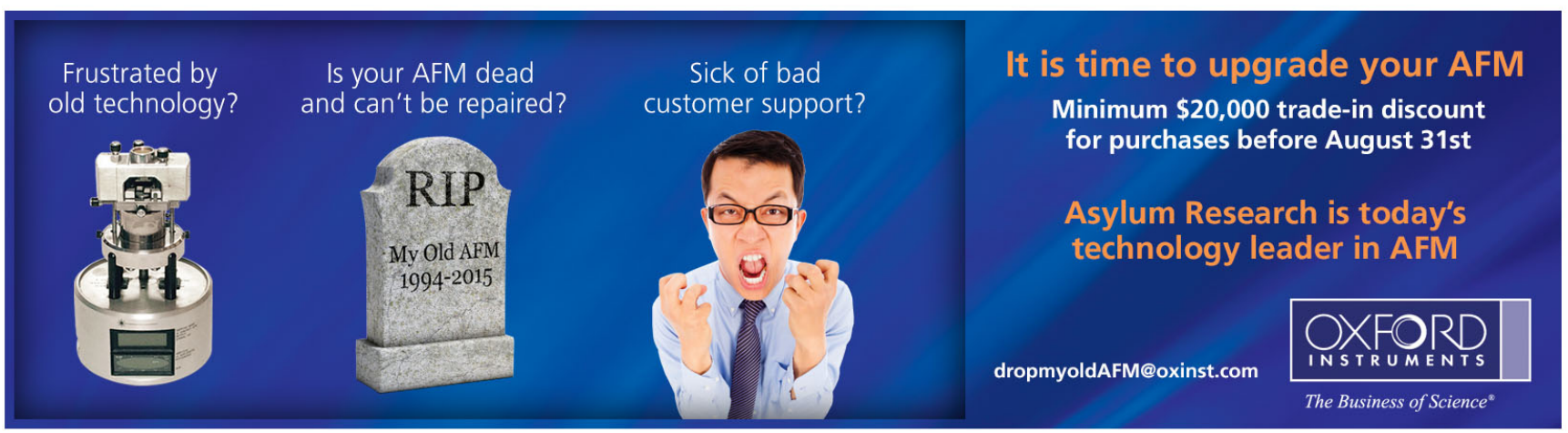




\title{
Control of thermal runaway in microwave resonant cavities
}

\author{
X. Wu and J. R. Thomas, Jr. ${ }^{\text {a) }}$ \\ Department of Mechanical Engineering, Virginia Polytechnic Institute and State University, Blacksburg, \\ Virginia 24061
}

W. A. Davis

Bradley Department of Electrical and Computer Engineering, Virginia Polytechnic Institute and State University, Blacksburg, Virginia 24061

(Received 6 March 2002; accepted for publication 28 June 2002)

\begin{abstract}
This article reports direct experimental evidence of the so-called "S curve" of temperature versus electrical field strength when materials with positive temperature dependence of dielectric loss are heated in a microwave resonant cavity applicator. A complete discussion of how the experimental results were achieved is presented. From the experimental results, we believe the $\mathrm{S}$ curve theory provides an incomplete explanation of thermal runaway in microwave heating. To understand microwave heating in a resonant cavity, cavity effects must be considered. To explain the experimental results, a theoretical model based on single-mode waveguide theory is developed. Finally, a method to control thermal runaway is described. (C) 2002 American Institute of Physics. [DOI: $10.1063 / 1.1501744]$
\end{abstract}

\section{INTRODUCTION}

Microwave heating is widely used in current industrial applications. The most unique characteristic of microwave heating is that the energy is absorbed volumetrically, producing internal heating. This is very different from conventional heating where the heat must diffuse in from the surface of the material. The power density absorbed by a material is related to the microwave electric field strength by the equation

$$
p_{\mathrm{dis}}=\omega \varepsilon^{\prime \prime}|E|^{2}=q_{\mathrm{abs}},
$$

where $\omega$ is the angular frequency, $|E|$ is the rms magnitude of the electric field strength in the material, and $\varepsilon^{\prime \prime}$ is the imaginary part of the complex permittivity of the material. From the electromagnetic field point of view, $p_{\text {dis }}$ is the power density dissipated in the materials. In the heat transfer equation, $q_{\text {abs }}$ represents the heat generation term.

The quantity $\varepsilon^{\prime \prime}$ plays an important role in the equation. Normally, $\varepsilon^{\prime \prime}$ depends upon the frequency of the microwave source and the temperature of the material. Since the frequency of the source is fixed in most applications, attention is focused on the temperature dependence of $\varepsilon^{\prime \prime}$. Several review articles describe some of the consequences of the temperature dependence of $\varepsilon^{\prime \prime} .^{1-3}$ If $\varepsilon^{\prime \prime}(T)$ increases abruptly above a certain temperature, the power density dissipated in the materials increases even when the electric field is unchanged, which leads to very rapid heating. On most occasions, this leads to the unwanted phenomenon of thermal runaway.

Numerous experimental reports of thermal runaway phenomena have been published. ${ }^{4-8}$ When input power level is below a critical input power, the steady state temperature of the material increases continuously with increasing input

${ }^{a)}$ Electronic mail: jthomas@vt.edu power. Yet the temperature of the processed material suddenly rises to a very high value as the input power level exceeds the critical input power. The temperature corresponding to the critical input power is called the critical temperature. The temperature of the material cannot be stabilized between the critical temperature and some higher value unless some control mechanisms are introduced.

Thermal runaway may be explained by the energy balance equation:

$$
\rho C_{p} \frac{d T}{d t}=q_{\mathrm{abs}}-q_{\mathrm{loss}},
$$

where $\rho, C_{p}$ are the density and specific heat of the material, respectively, and $q_{\text {loss }}$ is the rate of heat loss, which normally includes losses due to convection and radiation. Both $q_{\text {loss }}$ and $q_{\mathrm{abs}}$ are functions of temperature. If when starting from some critical temperature, $q_{\text {abs }}$ continues to exceed $q_{\text {loss }}$, thermal runaway will occur. Since $q_{\text {loss }}$ increases with $T$, the increase of $\varepsilon^{\prime \prime}$ with increasing $T$ provides a possible means for thermal runaway.

If the required processing temperature exceeds the maximum stable temperature, a control system must be employed, or the temperature will continue increasing until the material is damaged, which has occurred when sintering ceramic powder. ${ }^{6}$ In early experiments, when the desired temperature was reached, the power source was adjusted manually. ${ }^{6}$ These attempts sometimes led to success but, when materials are in a thermal runaway state, the temperature rise rate is rapid and temperature is highly sensitive to the incident power, making control highly problematic.

Beale et al. developed an automatic feedback control system which could regulate the material temperature above the critical value but also reported that the control of thermal runaway materials is not as accurate as with nonthermal runaway materials. ${ }^{9,10}$ Another approach to controlling microwave heating is based on the energy balance described by 


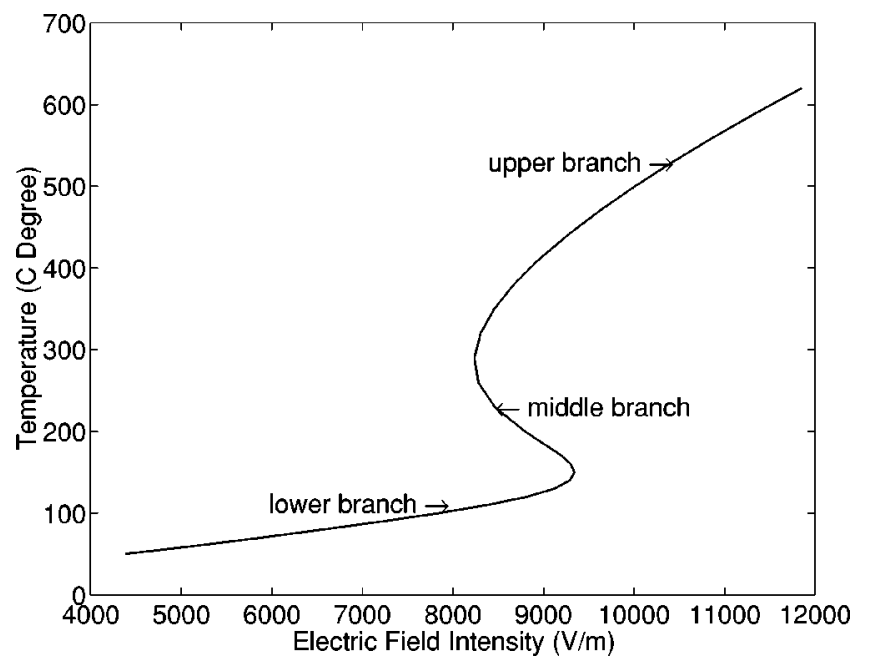

FIG. 1. S-shaped curve.

Thomas et al., ${ }^{11,12}$ who monitored and controlled the power absorbed by the sample. From an energy balance perspective, heat loss must be equal to power absorbed at steady state. Since heat loss is a monotonically increasing function of temperature, constant power absorbed by materials means a constant temperature; thus if power absorbed by materials can be held constant, thermal runaway can be avoided. However, to maintain constant absorbed power, a control system must be introduced.

A number of explanations of thermal runaway have been advanced. Kriegsmann developed a one-dimensional model for the heating of a ceramic slab by microwaves. ${ }^{13} \mathrm{He}$ found the important result that steady temperature as a function of the input microwave power gave an S-shaped response curve. A predicted S-shaped curve is illustrated in Fig. 1, where different electric field values represent different input power levels.

Every point on the curve represents a thermally balanced state. From this S-shaped curve, the dynamic response for different power levels can be derived. Based on the energy balance, Kriegsmann concluded that the middle branch of the S-shaped curve was unstable, so a temperature jump is possible, suggesting a plausible mechanism for thermal runaway control problems.

After Kriegsmann's initial work, some researchers examined thermal stability of materials with other shapes, such as a sphere and a cylindrical rod, heated by microwaves. ${ }^{14-17}$ All the analyses of thermal stability were based on the S-shaped curve. In all of this work, it was concluded that the instability of the middle branch of the S-shaped curve was the origin of thermal runaway.

In this article, we will show an experimentally observed S-shaped curve which includes the lower and middle branches. We find that the middle branch of the S-shaped curve is stable. To explain our experimental results, a singlemode waveguide model was developed, which includes both the material properties and cavity effects. We will see that cavity effects are very important in practical heating in resonant cavities. We also attempted to provide a method for control of thermal runaway based on the cavity effects.

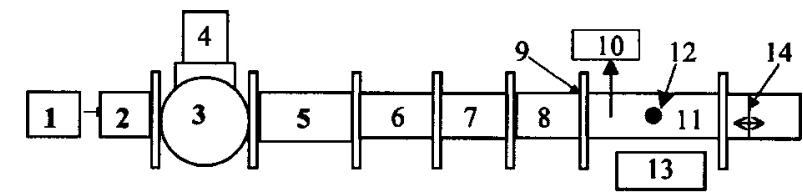

FIG. 2. Schematic of experimental setup (top view): (1) power source; (2) magnetron; (3) circulator; (4) water load; (5) waveguide extension; (6) four stub tuner; (7) impedance analyzer; (8) waveguide with wedge; (9) iris; (10) power meter for electric field measurement; (11) cavity; (12) sample; (13) pyrometer; (14) adjustable end-wall short.

\section{EXPERIMENT}

\section{A. Experimental setup}

The apparatus used for the experiments includes a power supply, remote microwave launcher (magnetron), circulator, water load, WR-284 copper waveguide cavity with coupling iris, waveguide, adjustable end-wall short, and power and temperature measurement equipment. The layout of the experimental components is shown in Fig. 2.

The microwave generator includes both the power supply and the remote launcher. The microwave system is powered by a Cober SM 1545D power supply and CWM-4-S magnetron, which can supply up to $3 \mathrm{~kW}$ to the applicator. The magnetron is water cooled and launches the $2.45 \mathrm{GHz}$ microwaves into a tapered WR-340 to WR-284 waveguide section. To protect the power generator from reflected power, the system is equipped with a circulator and water load.

To measure the power, a Homer impedance analyzer (by Cober) is introduced into the system. The impedance analyzer can measure incident power, reflection coefficient, and frequency.

The electric field strength in the cavity is measured using a power sensor and associated power meter. The power sensor is coupled to the electric field through a small probe inserted slightly into the cavity. The magnitude of the electric field strength is proportional to the square root of the measured power; the constant of proportionality is determined by measuring the probe power in an empty cavity with no iris and a termination load replacing the sliding short.

To precisely monitor the microwave heating process, two infrared radiation pyrometers (Heitronics KT 15.82D and KT 15.01D) were chosen. While the KT 15.82D is used to measure temperatures within a range of $0-500^{\circ} \mathrm{C}$, the $\mathrm{KT}$ 15.01D can measure temperatures within the range $350-2200{ }^{\circ} \mathrm{C}$. A radiation pyrometer measures the infrared radiation emitted by a surface; given the emissivity $\epsilon$ of the surface being measured, the temperature of the surface can be determined. The materials to be tested in this experimental program have emissivities that change significantly as a function of temperature. Varying the emissivity used by the pyrometer during an experiment is not practical since it must be done manually. Instead, the emissivity is set at 1.0 for the duration of the experiment, and the actual temperature is then determined from the measured temperature knowing the emissivity as a function of temperature for the material being heated, a process which is performed automatically by the data acquisition software. 


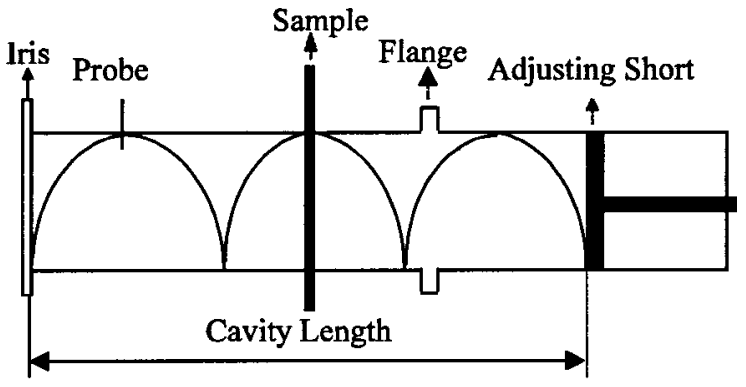

FIG. 3. Schematic of $\mathrm{TE}_{103}$ mode cavity.

The resonant cavity is designed to operate in the $\mathrm{TE}_{10 n}$ mode; using the adjustable short, the cavity was adjusted to operate near the cavity resonance of the $\mathrm{TE}_{103}$ mode for all of the experiments. The cavity includes three parts: the waveguide, input coupling iris, and adjustable short. The position of the short plane ranges from 1.1 to $10.7 \mathrm{~cm}$ if measured from the waveguide flange. The sample to be heated was placed in a port located $17.5 \mathrm{~cm}$ from the cavity aperture, which means it is always very close to an electric field peak for the $\mathrm{TE}_{103}$ mode as illustrated in Fig. 3 .

\section{B. Experimental results for $4.5 \mathrm{~mm}$ mullite rod}

The experimental results for a mullite rod with a diameter of $4.5 \mathrm{~mm}$ and a length of $30.5 \mathrm{~cm}$ are shown in Figs. 4 and 5 . Since the height of the cavity is only $3.4 \mathrm{~cm}$, only a small portion of the mullite is in the cavity. The output of the power generator is set to $600 \mathrm{~W}$, and is not changed during the experiment. The only adjustable component in the system is the position of the short plane, which changes the cavity length.

The experiment starts from a cavity length of $36.1 \mathrm{~cm}$ where the cavity is highly detuned. As Figs. 4 and 5 show, at the beginning of the experiment the temperature of the rod is ambient temperature. When the short is adjusted to decrease the length of the cavity, the temperature of the rod increases, as well as the electric field strength at the plane of the rod.

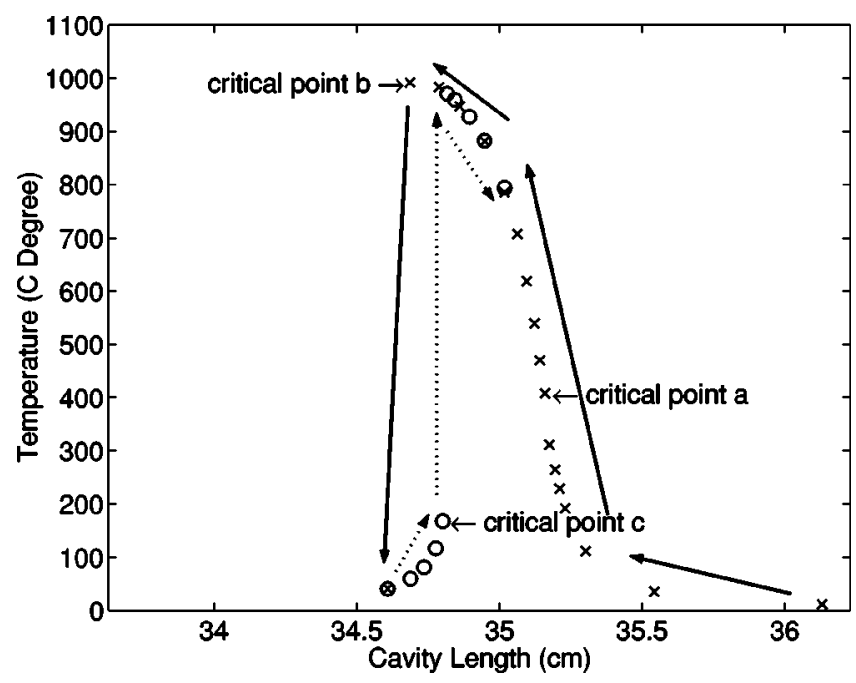

FIG. 4. Behavior of the rod temperature when the position of the short plane is adjusted.

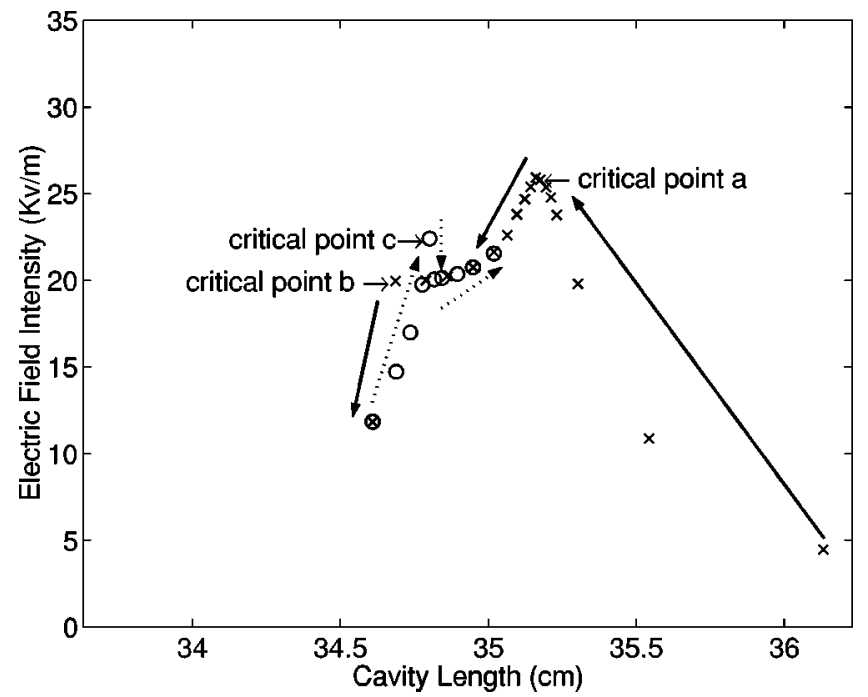

FIG. 5. Behavior of the electric field strength for the same experiment shown in Fig. 4.

Continuing to decrease the cavity length leads to critical point $a$, which is the point at which the maximum electric field strength is reached. From this point, the electric field strength always decreases independent of the direction of changes to the short plane position.

If we change the short plane position to increase the length of the cavity, then temperature and electric field strength decrease simultaneously to a new equilibrium point on the same curve. If we very gently adjust the short to further decrease the length of the cavity, the temperature of the rod will increase but the electric field strength will decrease due to the increased power absorption, leading to a new equilibrium point at higher temperature. If we continue decreasing the length of the cavity, the field in the cavity will collapse at critical point $b$. Both the temperature of the rod and the field intensity will drop dramatically, leading to equilibrium at a much lower temperature; this process is like reverse thermal runaway. All the points plotted in Figs. 4 and 5 represent stable states, and are denoted by the $\times$ symbol. The whole process path is denoted by the solid arrow.

To heat the rod from the lower temperature point, where the cavity length is $34.6 \mathrm{~cm}$, we must increase the cavity length. The temperature and the field will both increase along another curve which does not coincide with the original one. At critical point $c$, another electric field maximum is encountered. If the length of the cavity is increased from point $c$, a temperature excursion occurs. The temperature continues increasing and the field continues decreasing until an equilibrium point is reached that has much higher temperature and lower electric field strength. Notice that at the end of this process the field decreases rather than increases. If we continue to increase the length of the cavity, the temperature will decrease but the field will increase, and the equilibrium point curve will coincide with the original curve. All the stable points are denoted by " $\bigcirc$ " while the process path is denoted by a dashed arrow.

In Fig. 6, we collect the equilibrium points from Figs. 4 and 5 to plot temperature versus electric field strength. This 


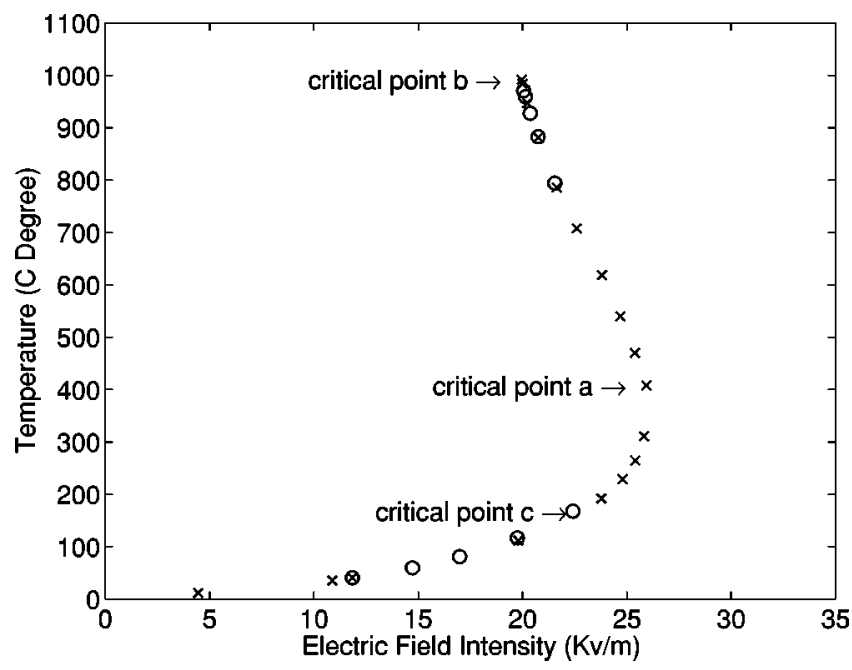

FIG. 6. Behavior of the electric field strength for the same experiment shown in Fig. 4.

clearly shows the lower and middle branches of the $\mathrm{S}$ curve.

\section{Typical heating processes}

There are four typical heating processes at constant power level. To illustrate the processes clearly, each is discussed and plotted on the $\mathrm{S}$ curve as follows.

\section{Decreasing the cavity length}

The process starts from an equilibrium point, and is illustrated in Fig. 7. When the cavity length is decreased, the electric field strength increases immediately while the temperature of the rod is nearly unchanged. When the rod heating begins, the cavity is detuned by the increasing dielectric loss of the rod resulting from the heating process. If the starting point is below the critical point $a$, this process will end at an absolutely stable point.

If the starting point is above the critical point $a$, the process will end at a relatively stable point. It is well known that all equilibrium points above the critical point $a$ are un-

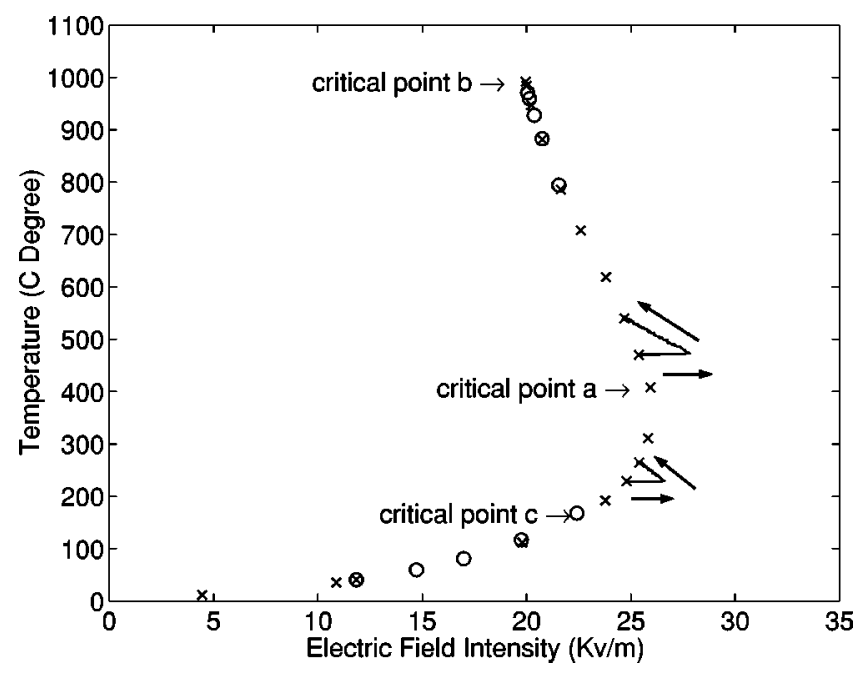

FIG. 7. Behavior of electric field strength and temperature for decreasing cavity length, illustrated for two different starting points.

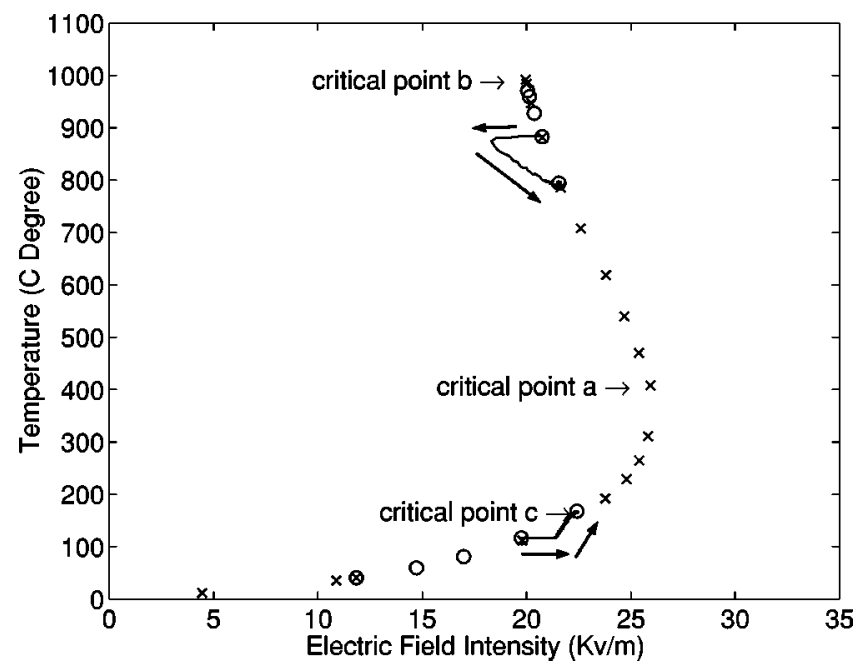

FIG. 8. Behavior of electric field strength and temperature for increasing cavity length, illustrated for two different starting points.

stable according to the $S$ curve theory. However, our experiment shows that the system is indeed stable if we heat the rod by decreasing the cavity length. The stability of the points on the middle branch of the $S$ curve will be discussed in Sec. IV A.

\section{Increasing the cavity length}

As illustrated in Fig. 8, when increasing the cavity length, the rod can be heated or cooled depending upon the starting point. If the starting point is below the critical point $\mathrm{c}$, the cavity will continue being tuned and the temperature will increase. If the starting point is above the critical point, the cavity is detuned first, but as the temperature increases, the cavity is moved toward the tuned state.

\section{Thermal excursion}

If the cavity length is increased from critical point $\mathrm{c}$, a rapid temperature excursion occurs in a rather complicated process. Although the temperature increases almost continuously, the field intensity does not vary monotonically. As illustrated in Fig. 9, the cavity is first highly tuned and the temperature increases rapidly. When the field intensity reaches its maximum value, the temperature increases dramatically during a very short time. In our experiments, the temperature increases more than $500^{\circ}$ in less than half a minute, causing the cavity to become detuned. At the end of the process, the temperature drops slightly to the equilibrium curve.

\section{Reverse thermal runaway}

If we decrease the length of cavity from the critical point $b$, a "reverse thermal runaway" occurs. The field strength decreases slightly, but the temperature drops precipitously, as illustrated in Fig. 10. 


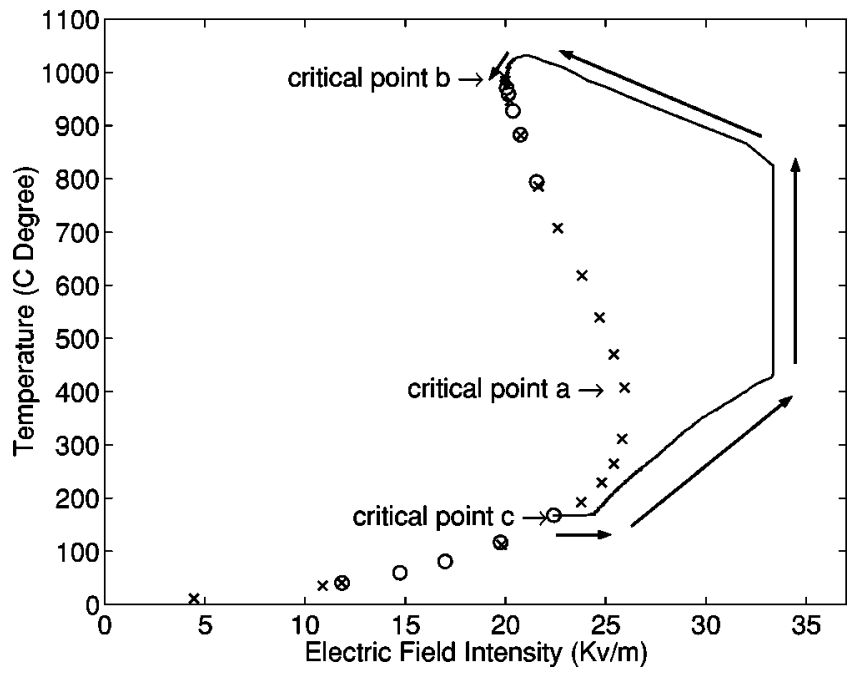

FIG. 9. Behavior of the electric field strength and temperature when the cavity length is increased from critical point $\mathrm{c}$. The temperature excursion mimics thermal runaway, but a stable equilibrium point is achieved at high temperature.

\section{THEORETICAL MODEL}

Contrary to most conclusions from theoretical modeling, we find that the middle branch of the $\mathrm{S}$ curve is at least locally stable for the mullite rods used in our experiments. To explain this phenomenon, a single-mode waveguide model was developed in which the cavity is simulated by an equivalent circuit as shown in Fig. 11. $I_{s}$ represents the source and $G_{s}$ represents the cavity loss, source conductance, and source generator. The heated sample is represented by $G_{s}+j B_{x}$ and $Z_{0}$ is the characteristic impedance of the waveguide for the $\mathrm{TE}_{10}$ mode. $l$ is the equivalent transmission line length.

Since the electric field at the sample is proportional to the voltage, it may be written as

$$
E=\frac{k I_{s}}{G_{s}+G_{x}+j\left(B_{x}-Y_{0} \cot \beta l\right)},
$$

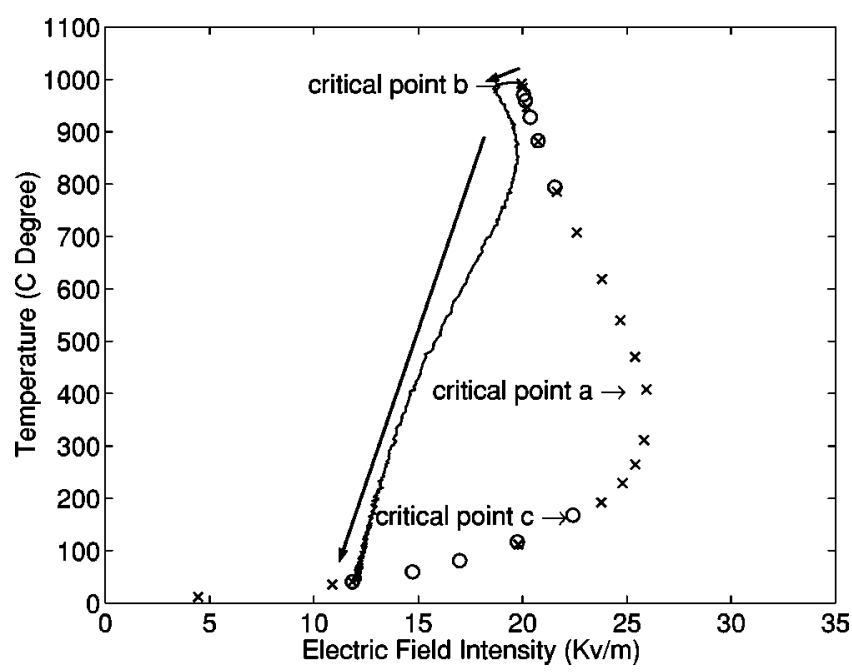

FIG. 10. Rapid temperature decrease following a decrease in cavity length from critical point $b$.

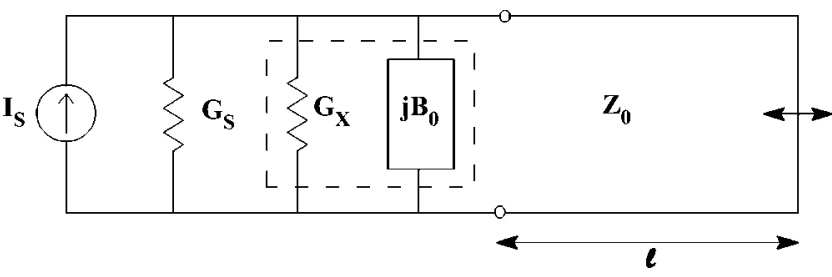

FIG. 11. Single-mode waveguide model for cavity.

where $\mathrm{k}$ is a proportionality constant. If all the quantities in the above expression are normalized by $G_{s}$, then the electric field at the sample becomes

$$
E=\frac{E_{0}}{1+g_{x}+j\left(b_{x}-y_{0} \cot \beta l\right)}
$$

where $E_{0}$ represents the peak electric field strength in the cavity without a sample. Since $g_{x}$ and $b_{x}$ represent the sample properties, they can be defined by

$$
g_{x}+j b_{x}=\omega c_{0}\left(\varepsilon_{r}^{\prime \prime}+j \varepsilon_{r}^{\prime}\right) .
$$

Thus,

$$
E=\frac{E_{0}}{1+\omega c_{0} \varepsilon_{r}^{\prime \prime}+j\left(\omega c_{0} \varepsilon_{r}^{\prime}-y_{0} \cot \beta l\right)},
$$

where $\beta$ is the wave number in the waveguide.

The two unknown numbers $c_{0}$ and $y_{0}$ represent the properties of the sample and waveguide. In the system, there are two parameters available to adjust, $E_{0}$ and $l$. During the experiment, the incident power is fixed, so the model is discussed under constant $E_{0}$. The electric field strength is a function of the equivalent transmission line length $l$ and the temperature through $\varepsilon_{r}^{\prime \prime}$ and $\varepsilon_{r}^{\prime}$. This relation is plotted on the $\mathrm{S}$ curve in the following paragraphs, where the equivalent transmission line length $l$ is a parameter.

If the incident power level is suitable, then the direction of movement of the short plane is important. The following discussion will be focused around a particular cavity length, $L_{0}$, which is the root of the equation $\omega c_{0} \varepsilon_{r}^{\prime}-y_{0} \cot (\beta l)$ $=0$. Equation (5) shows that the cavity is highly tuned at this cavity length.

If the cavity is highly detuned because the cavity length is too long, $l \gg L_{0}$, decreasing the cavity length tends to tune the cavity and the temperature of the sample increases. This is illustrated in Fig. 12, where plots of Eq. (5) for different cavity lengths $l$ are superimposed on a plot of the experimental points shown in Fig. 6 . The field strength in the cavity must lie on one of the solid curves, while the temperature which also satisfies the energy balance must lie on a line connecting the experimental points. Thus, we can see how changing the cavity length moves the equilibrium point along the experimental curve.

If the cavity length is decreased from $L_{1}$ through $L_{2}$ to $L_{3}$ as shown in Fig. 12, the point of intersection with the experimental curve will eventually pass the critical point $a$. Near this point, if we shorten the length of the cavity from point $s$, the electric field strength will initially increase and the temperature will be unchanged. In the E-T plane, this state is represented by point $m$. Starting from point $m$, the 


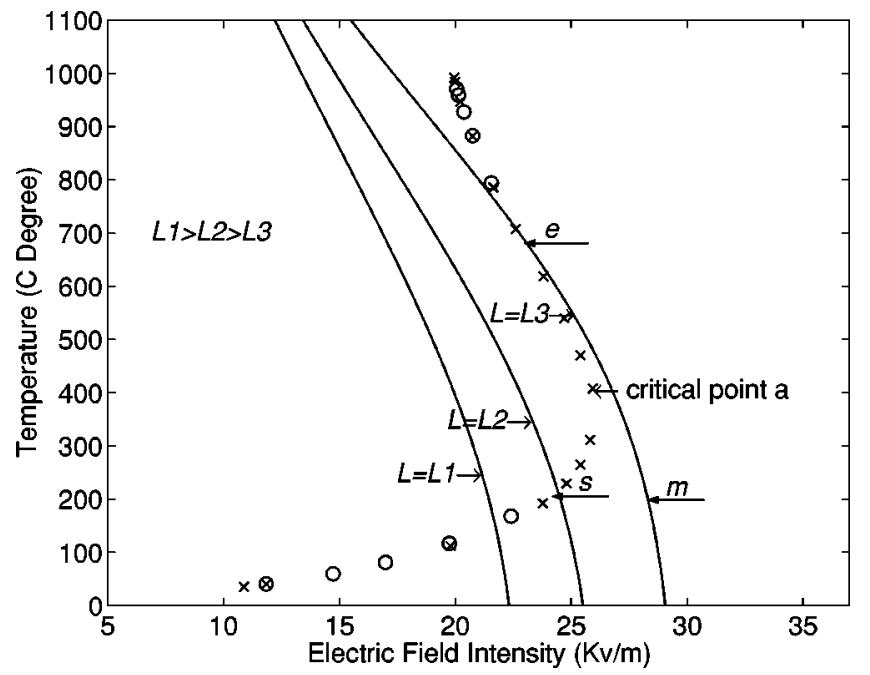

FIG. 12. Behavior of electric field strength and temperature for decreasing cavity length.

energy balance will force the temperature of the sample to rise. This temperature increase causes the dielectric properties of the sample to change, which then detunes the cavity; this can be derived from Eq. (5). The electric field strength is a monotonically decreasing function of temperature if $l$ $>L_{0}$. Although the cavity is detuned, the absorbed energy is still larger than the heat loss, so the temperature continues increasing until it again intersects the $\mathrm{S}$ curve at point $e$, as illustrated in Fig. 12.

If the cavity is highly detuned because the length of the cavity is too short, $l \ll L_{0}$, we must increase the cavity length to tune the cavity to heat the sample. This process is initially stable, but as the critical point $c$ is approached, the process may become unstable. This is shown in Fig. 13. If the length of the cavity is increased from point $s$, the electric field strength will initially increase and the temperature will be unchanged. This step is the same as decreasing the cavity length and is also illustrated by point $m$. At this point, the term $\omega c_{0} \varepsilon_{r}^{\prime}-y_{0} \cot \beta l$ in Eq. (5) is negative since $L_{6}<L_{0}$.

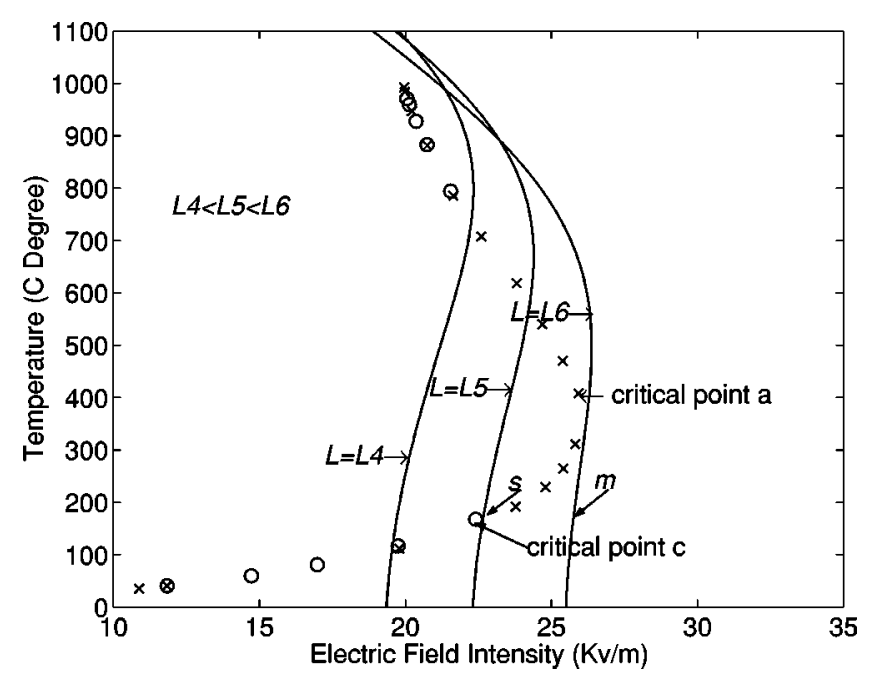

FIG. 13. Behavior of electric field strength and temperature for increasing cavity length.

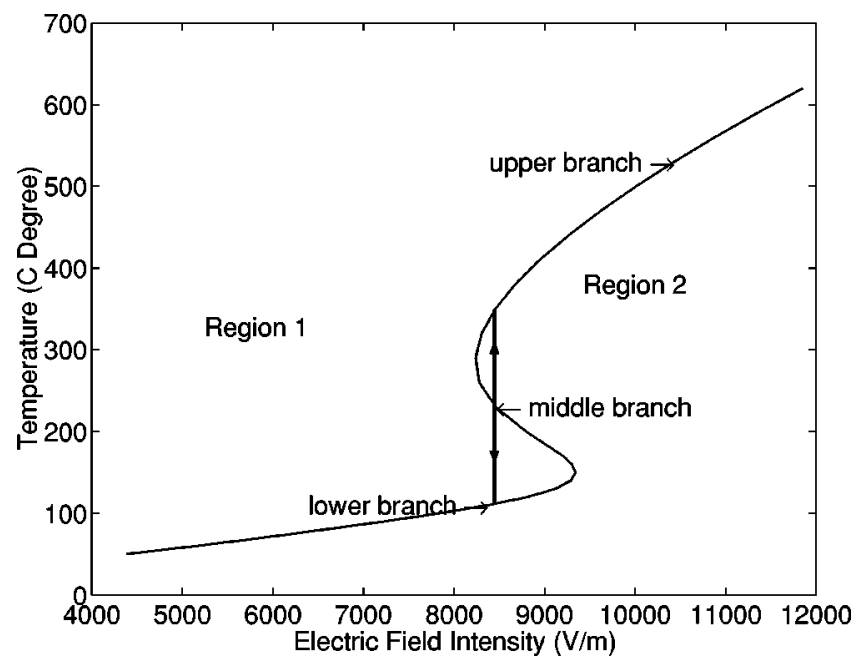

FIG. 14. Stability analysis using S-shaped curve only.

Starting from point $m$, the energy balance will force the temperature of the sample to rise. Equation (5), shows that the cavity is tuned rather than detuned until the electric field strength reaches its maximum value. This provides a possible path for the electric field strength versus temperature curve to circumvent the $\mathrm{S}$ curve at the temperature around critical point $a$. This process may end at a higher temperature or thermal runaway may occur.

\section{DISCUSSION}

\section{A. Stable or unstable?}

Previous authors used only the S-shaped curve to analyze stability. ${ }^{13}$ This is illustrated by Fig. 14 . The whole E-T plane is divided by the S-shaped curve into two regions. In region $1, q_{\text {abs }}$ is less than $q_{\text {loss }}$, thus the temperature tends to decrease while in region $2, q_{\text {abs }}$ is larger than $q_{\text {loss }}$, and the temperature tends to increase. This leads to the conclusion that the middle branch of the S-shaped curve must be unstable since the system cannot tolerate any disturbance from equilibrium. Since this conclusion is based on an energy balance, it is considered unassailable, but is based on the implicit assumption that the electric field is constant when the temperature is changing. This is clearly not valid for microwave heating in the cavity.

In the cavity, the variation of the temperature of the materials changes the electric field. The actual heating process is illustrated in Fig. 15.

Around the middle branch of the S-shaped curve, if the processing point is in region 1 , the temperature must decrease since $q_{\text {abs }}$ is less than $q_{\text {loss }}$. At the same time, it is apparent from Eq. (5) that the electric field strength must increase, so the processing point returns to the equilibrium point on the S-shaped curve. If the processing point is in region 2, it also must return to the equilibrium point. Since the response time of the electromagnetic field is much smaller than that of the thermal process, the middle branch of the $\mathrm{S}$-shaped curve is stable. Viewed from this perspective, there is no difference between the lower or middle branch of the $\mathrm{S}$ curve. 


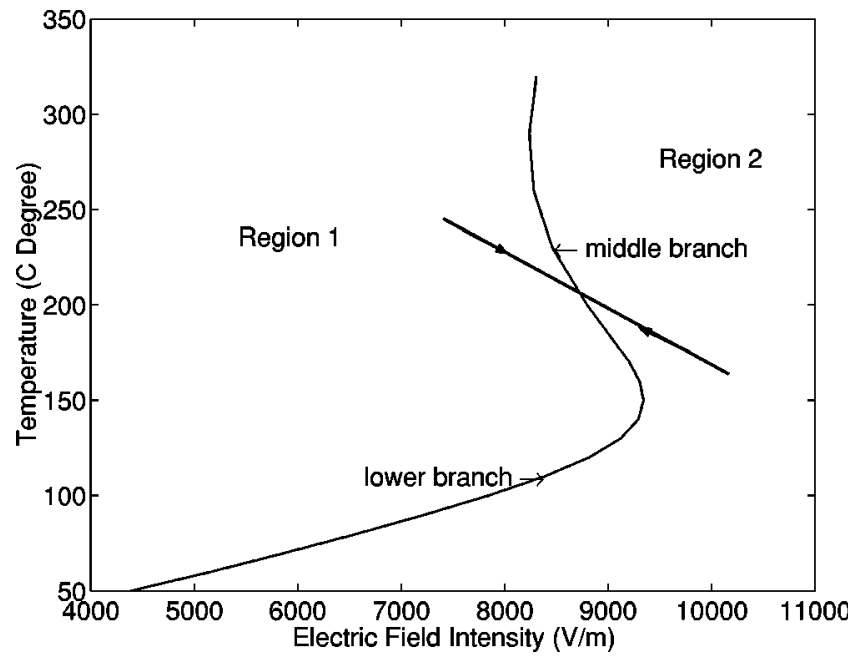

FIG. 15. Stability analysis using both S-shaped curve and cavity effects.

\section{B. Control of thermal runaway}

From the experimental results and the theoretical model, it is clear that when investigating stability or instability of a system, all parameters must be considered. In our system, the electric field acts as an automatic feedback control if adjusting the short plane in the correct way. Since the response time of the electromagnetic field is so small, we believe this is a good control system. Figure 16 shows the temperature versus time when the mullite rod was heated from 790 to $990^{\circ} \mathrm{C}$. This temperature change was produced simply by changing the cavity length, after which the system evolved automatically. For this system, $990^{\circ} \mathrm{C}$ is on the middle branch of the S-shaped curve.

\section{CONCLUSIONS}

In our experiments on heating of ceramic rods in a $\mathrm{TE}_{103}$ cavity, we discovered three clearly identifiable critical tuning points, and explored the heating behavior resulting from changes of cavity length from each of these tuning points. Two branches of the well-known $\mathrm{S}$ curve first identified by Kriegsmann have been experimentally observed.

The stability of the system is discussed on the basis of both energy balance and cavity effects. Contrary to most conclusions from theoretical modeling, we find that the middle branch of the S-shaped curve is stable. Viewed from the point of view of stability, we conclude there is no difference between the lower and middle branches of the S-shaped

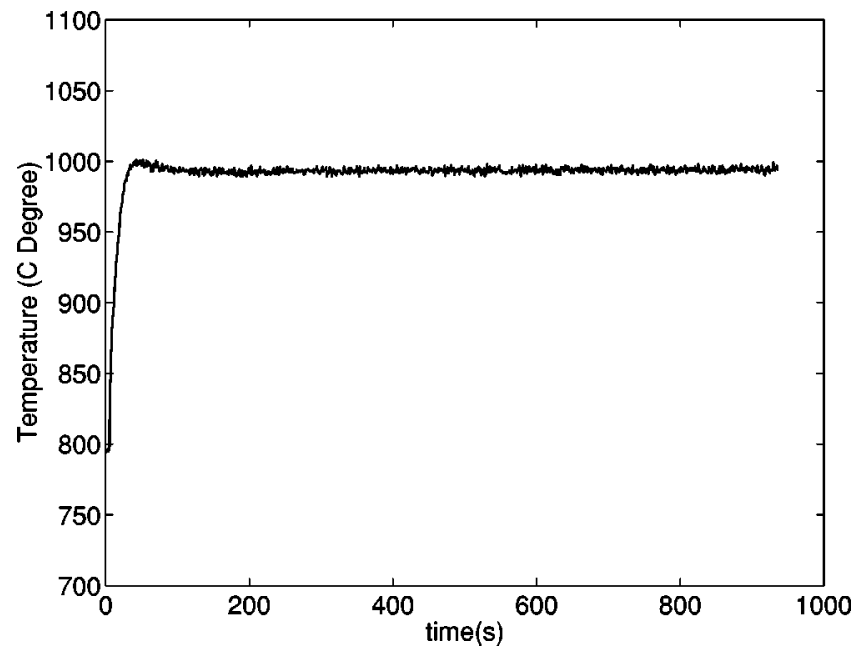

FIG. 16. Temperature profile heated from 790 to $990{ }^{\circ} \mathrm{C}$.

curve. Thermal runaway in microwave heating in the cavity is due to the combination of the energy balance and cavity effects. Adjusting the short plane correctly can avoid thermal runaway, at least for mullite heated in a $\mathrm{TE}_{103}$ cavity.

${ }^{1}$ D. E. Clark and W. H. Sutton, Annu. Rev. Mater. Sci. 26, 299 (1996).

${ }^{2}$ W. H. Sutton, Am. Ceram. Soc. Bull. 68, 376 (1989).

${ }^{3}$ W. H. Sutton, Ceram. Trans. 36, 3 (1993).

${ }^{4}$ D. L. Johnson, Ceram. Trans. 21, 283 (1991).

${ }^{5}$ S. L. McGill, J. W. Walkiewicz, and G. A. Smyres, in Microwave Processing of Materials, edited by W. H. Sutton, M. H. Brooks, and I. J. Chabinsky, MRS Symposia Proceedings No. 124 (Materials Research Society, Pittsburgh, 1988), pp. 247-252.

${ }^{6}$ Y. L. Tian, D. L. Johnson, and M. E. Brodwin, Proceedings of the First International Conference on Ceramic Powder Processing Science, Orlando, FL, Nov. 1987.

${ }^{7}$ Y. L. Tian, Ceram. Trans. 21, 283 (1991)

${ }^{8}$ X. Zhang, D. O. Hayward, and D. M. P. Mingos, Ind. Eng. Chem. Res. 40, 2810 (2001).

${ }^{9}$ G. O. Beale and F. J. Arteaga, Mater. Res. Soc. Symp. Proc. 269, 265 (1992).

${ }^{10}$ G. O. Beale and M. Li, IEEE Trans. Ind. Electron. 44, 124 (1997).

${ }^{11}$ J. R. Thomas, Jr., C. C. Goodson, C. H. Stern, M. Jackson, and G. J. Vogt, J. Microw. Power Electromagn. Energy 33, 113 (1998).

${ }^{12}$ J. R. Thomas, Jr., J. M. Curtis, Jr., C. H. Stern, and W. A. Davis, Ceram. Trans. 111, 345 (2000).

${ }^{13}$ G. A. Kriegsmann, J. Appl. Phys. 71, 1960 (1992).

${ }^{14}$ N. Gupta, V. Midha, V. Balakotaiah, and D. J. Economou, J. Electrochem. Soc. 146, 4659 (1999).

${ }^{15}$ H. W. Jackson, M. Barmatz, and P. Wagner, Ceram. Trans. 36, 189 (1993).

${ }^{16}$ M. S. Spotz, D. J. Skamser, and D. L. Johnson, J. Am. Ceram. Soc. 78, 1041 (1995)

${ }^{17}$ C. A. Vriezinga, J. Appl. Phys. 85, 3774 (1999). 